\title{
Numerical schemes for networks of hyperbolic conservation laws
}

\author{
Raul Borsche ${ }^{\mathrm{a}}$ \\ ${ }^{a}$ Erwin Schrödinger Straße, TU Kaiserslautern, Building 48, 67663 Kaiserslautern, \\ Germany
}

\begin{abstract}
In this paper we propose a procedure to extend classical numerical schemes for hyperbolic conservation laws to networks of hyperbolic conservation laws. At the junctions of the network we solve the given coupling conditions and minimize the contributions of the outgoing numerical waves. This flexible procedure allows us to also use central schemes at the junctions. Several numerical examples are considered to investigate the performance of this new approach compared to the common Godunov solver and exact solutions.
\end{abstract}

\section{Introduction}

Networks of hyperbolic balance laws occur in many applications, e.g. gas $[1,9]$, sewer $[17,15]$ and road networks $[7,14,6]$ or the human circulatory system $[13,19]$. Most of these applications require fast, stable and accurate solvers in order to approximate the solution on such networks numerically. Inside each edge of the network classical solvers for hyperbolic conservation laws can be used $[18,21]$. At the junctions suitable coupling conditions are imposed. Numerical methods solving these coupling conditions should only use waves entering the domain. In this context often a Godunov-type scheme is chosen in combination with a nonlinear Newton-type solver for the algebraic coupling conditions [5, 1 , 17]. The Godunov method requires a very detailed knowledge of the solution of the Riemann problem, which in some cases may be hard or impossible to construct [10].

The present paper introduces a procedure to extend most of the classical solvers for hyperbolic conservation laws onto junctions of networks. The procedure involves two main aspects. At first the coupling conditions at the junction have to be solved. These involve the states at the interface of the boundary, i.e. suitable approximations of these states have to be specified. Secondly the fluxes at the node should only result from waves entering the domain. As for central solvers it is impossible to strictly separate the orientation of the particular waves, we aim to minimize the contributions of the exiting waves. Both tasks are considered simultaneously as a constrained nonlinear optimization problem 
and can be solved with standard techniques. The resulting scheme on the complete network is conservative up to the precision of the nonlinear solver used for solving the coupling conditions.

This paper is organized as follows: In the first part we fix the notation and describe a general procedure for numerical solvers at a junction. In section 4 this method is applied to the Godunov, Roe, Lax-Friedrichs and FORCE scheme. Finally the properties of these different solvers are investigated in several numerical examples.

\section{Networks of hyperbolic conservation laws}

Networks of hyperbolic conservation laws consist of several edges and nodes. On each edge the transport of the considered quantities $u$ is governed by a hyperbolic conservation law. At the nodes these quantities are connected by so called coupling conditions. In general it is sufficient to study only a single junction due to the finite speed of propagation of information. Further w.l.o.g. we assume all edges to be orientated away from the junction. Thus we can formulate the problem as

$$
\begin{array}{ll}
\partial_{t} u^{k}(t, x)+\partial_{x} f^{k}\left(u^{k}(t, x)\right)=0 & x \in[0, \infty), t \geq 0, \quad k=1, \ldots, m \\
\Phi\left(u^{1}(t, 0+), \ldots, u^{n}(t, 0+)\right)=0 & t \geq 0 .
\end{array}
$$

where $u^{k}$ are the conserved quantities on the edge $k$ and $f^{k}$ the corresponding flux function. The coupling conditions $\Phi:\left(\mathbb{R}^{n}\right)^{k} \rightarrow \mathbb{R}^{c}$ connect the states at the left ends of the spatial domain for all times $t$. The number of coupling conditions $c$ depends on the scenarios considered and has to be chosen equal to the number of characteristics entering the domain. Under suitable assumptions the problem $(1),(2)$ is well-posed [11]. Here we only consider hyperbolic conservation laws, but the presented procedure can be easily extended to networks of balance laws.

\section{Numerical Solver}

Many applications for networks of hyperbolic conservation laws require accurate numerical schemes to approximate the exact solutions. For single hyperbolic conservation laws a huge variety of solvers is available [18, 21]. Most of them are based on the conservative update formula

$$
u_{i}^{j+1}=u_{i}^{j}-\frac{\Delta t}{\Delta x}\left(F_{i+1 / 2}-F_{i-1 / 2}\right),
$$

on an equidistant grid with spacing $\Delta x$. In the context of finite volume schemes the cell average $u_{i}^{j}$ of the cell $\left[x_{i-1 / 2}, x_{i+1 / 2}\right]$ at time $t^{j}$ is updated by the numerical fluxes $F_{i+1 / 2}=F\left(u_{i}, u_{i+1}\right)$ to the state $u_{i}^{j+1}$ at $t^{j+1}=t^{j}+\Delta t$.

Alternatively equation (3) can be reformulated using the so called fluctuations $[18,21]$ to

$$
u_{i}^{j+1}=u_{i}^{j}-\frac{\Delta t}{\Delta x}\left(D_{i+1 / 2}^{-}+D_{i-1 / 2}^{+}\right)
$$


where we define the fluctuations $D^{ \pm}$as

$$
D_{i+1 / 2}^{-}=F_{i+1 / 2}-f\left(u_{i}^{j}\right) \quad \text { and } \quad D_{i-1 / 2}^{+}=f\left(u_{i}^{j}\right)-F_{i-1 / 2} .
$$

The term $D_{i+1 / 2}^{-}$accumulates all contributions of backward going waves at the interface $x_{i+1 / 2}$, whereas $D_{i-1 / 2}^{+}$contains all forward going waves at the interface at $x_{i-1 / 2}$.

In contrast to this classical situation only very few solvers have been developed for junctions of networks of hyperbolic conservation laws, e.g. [1, 9, 13, 5 , 16]. A solver at the junction should focus on two main properties

1) The states at the interface at $x=0$ solve the coupling conditions.

2) The solver should only use waves entering the domain.

In fact the second property (7) is a natural consequence of the first one (6), since the states at the interfaces can only be connected with the states inside the domain by right going waves. Both aspects arise naturally from the construction in the well-posedness result of the coupling conditions [11].

For numerical methods it is often convenient to use so called ghost-cells at the boundaries $[18,21]$. Here we focus on first order schemes, i.e. we just need one ghost cell. A procedure to extend a first order method to higher orders has been developed in [5]. In the following we will use the notation depicted in figure 1. $u_{L}^{k}$ denotes the state in the ghost cell of edge $k, u_{R}^{k}$ is the value in

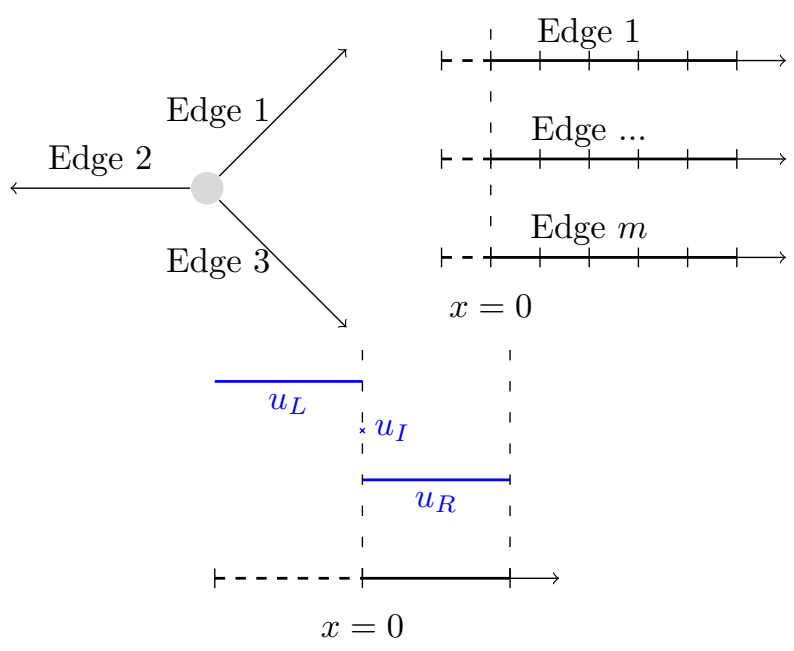

Figure 1: Notation at the interface.

the first cell inside the domain and $u_{I}^{k}$ is the approximate value at the interface. In the following we will drop the superscript index $j$ denoting time, as well the superscript index $k$ corresponding to the edges if possible. 
Thus in order to construct a numerical method for networks of hyperbolic conservation laws we just have to define a procedure how to determine the values in the ghost cells $u_{L}^{k}$, i.e. we have to specify the $m \cdot n$ unknowns in the ghost cells.

\subsection{Solving the coupling conditions}

The main aspect of the solution at the junction is that the coupling conditions (2) are satisfied. When using a numerical scheme only approximate values of $u(t, 0+)$ are available, i.e. we impose

$$
\Phi\left(u_{I}^{1}, \ldots, u_{I}^{n}\right)=0
$$

at every discrete time level. The approximation of $u_{I}$ should represent the state at the interface according to the underlying numerical scheme which is used. Thus the simple average $u_{I}=\frac{1}{2}\left(u_{R}+u_{L}\right)$ is not a correct choice in general. Examples considering some common schemes are provided in section 4 .

\subsubsection{Conservation of mass}

There is one particular but common situation when the above procedure should be modified. Often the coupling conditions state that some of the quantities which are governed by the conservation laws should also be conserved across the junction, e.g. the conservation of mass. Assume that the quantity $(u)_{\tilde{c}}$ is to be conserved, where $\tilde{c}$ is some index of the components of $u$. Then the coupling conditions contain the equation

$$
\sum_{k=1}^{m}\left(f^{k}\left(u^{k}(t, 0+)\right)\right)_{\tilde{c}}=0 .
$$

This equation can be easily transfered to the numerical scheme by requiring

$$
\sum_{k=1}^{m}\left(F^{k}\left(u_{L}, u_{R}\right)\right)_{\tilde{c}}=0
$$

instead of $\sum_{k=1}^{m}\left(f^{k}\left(u_{I}^{k}\right)\right)_{\tilde{c}}=0$. In fact the total mass of the quantity $(u)_{\tilde{c}}$ is conserved if there is no outflow at the right ends, i.e. $\left(F_{N+1 / 2}^{k}\right) \tilde{c}=0$, and (10) holds

$$
\begin{aligned}
\sum_{k=1}^{m} \sum_{i=1}^{N}\left(u_{i}^{j+1}\right)_{\tilde{c}} & =\sum_{k=1}^{m}\left(\sum_{i=1}^{N}\left(u_{i}^{j}-\frac{\Delta t}{\Delta x}\left(F_{i+1 / 2}^{k}-F_{i-1 / 2}^{k}\right)\right)\right)_{\tilde{c}} \\
& =\sum_{k=1}^{m}\left(\sum_{i=1}^{N}\left(u_{i}^{j}\right)_{\tilde{c}}-\frac{\Delta t}{\Delta x}\left(F_{N+1 / 2}^{k}-F_{1 / 2}^{k}\right)_{\tilde{c}}\right) \\
& =\sum_{k=1}^{m} \sum_{i=1}^{N}\left(u_{i}^{j}\right)_{\tilde{c}}+\frac{\Delta t}{\Delta x} \sum_{k=1}^{m}\left(F^{k}\left(u_{L}, u_{R}\right)\right)_{\tilde{c}}=\sum_{k=1}^{m} \sum_{i=1}^{N}\left(u_{i}^{j}\right)_{\tilde{c}} .
\end{aligned}
$$

Thus whenever the conservation of some quantity is desired we replace its part (9) of (8) by (10). 


\subsection{Preferring right going waves}

In general it is not sufficient to state the coupling conditions to uniquely determine the states in the ghost cells, since we just have $m$ nonlinear equations for $m \cdot n$ unknowns.

As stated in (7), we are only interested in waves entering the domain, since we can not track left going waves. Thus we are looking for states $u_{L}$ such that $F\left(u_{L}, u_{R}\right)$ contains only right going waves. Alternatively this can be formulated in terms of the fluctuations (5)

$$
D^{-}\left(u_{L}^{k}, u_{R}^{k}\right)=0 .
$$

Unfortunately for some solvers it is not possible to find states $u_{L}^{k}$ which simultaneously solve the coupling conditions (8) and fulfill (11) exactly, as discussed e.g. in section 4.3 for the Lax-Friedrichs scheme. As we do not want to modify the coupling conditions we have to relax (11) to

$$
\min _{u_{L} \in \mathbb{R}^{m \cdot n}} \sum_{k=1}^{m}\left\|D^{-}\left(u_{L}^{k}, u_{R}^{k}\right)\right\|_{2} .
$$

Clearly a solution of (11) is a minimizer of (12). Thus the combination of both properties (8) and (12) leads to the following nonlinear constraint minimization problem at the junction

$$
\begin{aligned}
& \min _{u_{L} \in \mathbb{R}^{m \cdot n}} \sum_{k=1}^{m}\left\|D^{-}\left(u_{L}^{k}, u_{R}^{k}\right)\right\|_{2} \\
& \text { s.t. } \Phi\left(u_{I}\left(u_{L}^{1}, u_{R}^{1}\right), \ldots, u_{I}\left(u_{L}^{m}, u_{R}^{m}\right)\right)=0 .
\end{aligned}
$$

A procedure how to approximate such $u_{L} \mathrm{~s}$ numerically is described in section 5 .

\section{Examples of numerical solvers}

In this section we discuss the above procedure on the basis of four classical solvers. We do not present a detailed formulation of the original numerical methods and refer to the standard literature $[18,21]$ for more information. The above procedure is not restricted to the following choices, but by the following examples we aim to cover the main types of classical solvers available.

\subsection{Godunov solver}

In the context of networks of hyperbolic conservation laws, the Godunov method is the most used solver at the junctions. In the following we will see that its particular usage, e.g. in [5, 14, 20], coincides with the above procedure.

In the Godunov method the inter-cell flux is defined by evaluating the exact solution of the Riemann problem at the interface $u_{G}$, i.e. $F\left(u_{L}, u_{R}\right)=f\left(u_{G}\right)$. Since we are considering only cases without transsonic waves, the state $u_{G}$ is 
a constant intermediate states of the exact solution to the Riemann problem. Thus $u_{G}$ can be determined by concatenating the Lax-Curves of all right going waves. In this context the choice of $u_{I}$ in (8) is naturally provided by $u_{I}=u_{G}$, as $u_{G}$ is locally the exact state at the interface. For the Godunov method equation (9) and (10) coincide. The constraint to only use right going waves leads immediately to $u_{L}=u_{G}$. In this case equation (11) is fulfilled exactly and thus also (12).

For the Godunov solver the numerical effort of the above procedure can be reduced by directly inserting the concatenations of the Lax-Curves of the right going waves into the coupling conditions. This leads to a single nonlinear system to be solved and does not involve any minimization procedure.

\subsection{Roe solver}

The Roe-flux is determined on the basis of the so called Roe-linearization $\hat{u}$, which depends nonlinearly on the states $u_{R}$ and $u_{L}$.. It is used to formulate a linear Riemann problem with waves $\hat{\mathcal{W}}_{l}$ of strength $\hat{\alpha}_{l}$ and speed $\hat{\lambda}_{l}$. The flux of the Roe method can be written as

$$
F_{R o e}\left(u_{L}, u_{R}\right)=f\left(u_{L}\right)+\sum_{l: \hat{\lambda}_{l}<0} \hat{\alpha}_{l} \hat{\lambda}_{l} \hat{\mathcal{W}}_{l} .
$$

Thus the Roe solver provides detailed information about the wave structure at the interface. The approximation of the state at the interface is directly given by the solution of the linear problem at this position

$$
u_{I}=u_{R}-\sum_{l: \hat{\lambda}_{l}>0} \hat{\alpha}_{l} \hat{\mathcal{W}}_{l} .
$$

Alternatively an approximation used for central schemes (15) can be used, which leads to similar results.

Using the Roe flux (13) in (11) we obtain

$$
0=D^{-}\left(u_{L}, u_{R}\right)=\sum_{l: \hat{\lambda}_{l}<0} \hat{\alpha}_{l} \hat{\lambda}_{l} \hat{\mathcal{W}}_{l}
$$

This equation appears to be the fluctuation of a linear system, but determining $u_{L}$ such that the above condition (14) holds is a nonlinear task, since in general the involved variables depend nonlinearly on $u_{L}$. Nevertheless under mild assumptions it appears to be possible to fulfill (11) exactly. The relation (14) holds if $\hat{\alpha}_{l}=0$ for all $l$ s.t. $\hat{\lambda}_{l}<0$, which are exactly $m \cdot n-c$ scalar equations. Together with the coupling conditions (8) we obtain $m \cdot n$ nonlinear equations for $m \cdot n$ unknowns. Further in a linearization around $u_{R}$ we obtain a uniquely solvable system, since the set of the eigenvectors is linearly independent.

In order to illustrate the solvability of this system of equations we consider the shallow water equations (21) in the case of subcritical flow. The strength of 
the 1-wave is given by

$$
\hat{\alpha}_{1}=\frac{\left(h_{R}-h_{L}\right) \hat{\lambda}_{2}-\left(q_{R}-q_{L}\right)}{2 \sqrt{\hat{h}}} .
$$

Since in the Roe-linearization the waves correspond to the Hugoniot loci of the exact solution we obtain by setting $\hat{\alpha}_{1}=0$

$$
\begin{aligned}
& \frac{\sqrt{h_{R}} \frac{q_{R}}{h_{R}}+\sqrt{h_{L}} \frac{q_{L}}{h_{L}}}{\sqrt{h_{R}}+\sqrt{h_{L}}}+\sqrt{\frac{1}{2}\left(h_{R}+h_{L}\right)}=\frac{q_{R}-q_{L}}{h_{R}-h_{L}} \\
& \Rightarrow q_{L}=\frac{h_{L}}{h_{R}} q_{R}+\left(h_{L}-h_{R}\right) \sqrt{\frac{h_{L}}{h_{R}} \frac{1}{2}\left(h_{R}+h_{L}\right)},
\end{aligned}
$$

which is just the Hugoniot loci corresponding to the rightgoing 2-wave. At the point $h_{L}=h_{R}$ this curve has as tangental the eigen vector $r_{2}$ corresponding to the 2 -wave $[18,21]$. Thus in combination with the well-posedness condition of the coupling conditions [11]

$$
\operatorname{det}\left[\partial_{u^{1}} \Phi r_{2}^{1}, \ldots, \partial_{u^{m}} \Phi r_{2}^{m}\right] \neq 0
$$

we can locally state the existance of the desired states $u_{L}^{k}$.
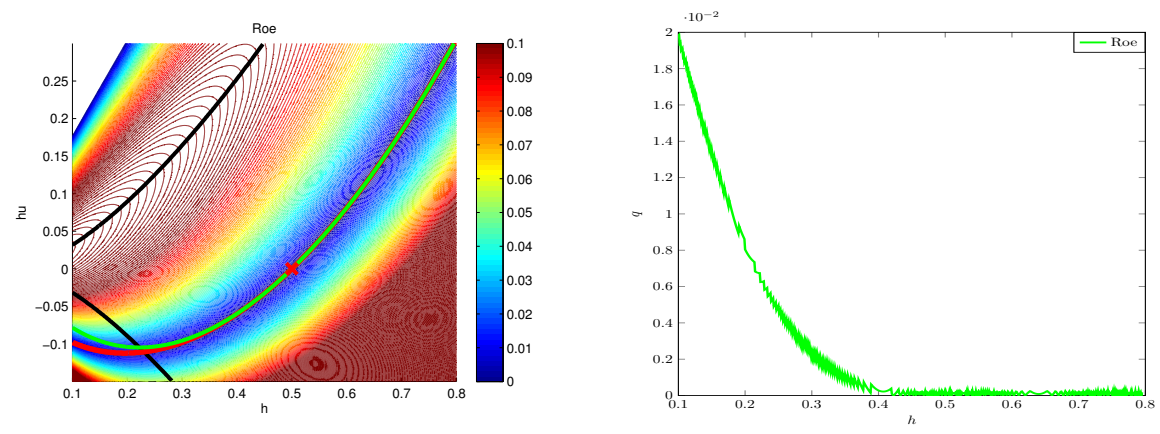

Figure 2: Roe method: Left: The value of $\left\|D^{-}\left(u_{L}, u_{R}\right)\right\|_{2}$ for $u_{R}=\left(\frac{1}{2}, 0\right)^{T}$ and varying $u_{L}$. Right: Deviation of the magenta curve from the exact Lax-Curve (green).

In order to illustrate the deviation from the exact Lax-Curve in case of the shallow water equations we depict the value of $\left\|D^{-}\left(u_{L}, u_{R}\right)\right\|_{2}$ for a fixed value of $u_{R}=\left(\frac{1}{2}, 0\right)^{T}$ and varying $u_{L}$ in the left part of figure 2 . The green line draws the exact Lax-Curve and the magenta curve indicates the minimum w.r.t. $q$ for each value of $h_{L}$. The black lines indicate the transsonic borders in the state space. As we have shown before the two curves coincide in case of a shock wave and differ only little in case of a rarefaction wave. On the right side of 2 the distance of the magenta curve to the exact Lax-curve is plotted. Thus in case of the Roe method we expect the solution of (11) to be a good approximation of the exact state at the junction. 


\subsection{Lax-Friedrichs solver}

The Lax-Friedrichs scheme is one of the simplest solvers available, but nevertheless it can refer to a certain popularity $[18,21]$. As it is a so called central solver we do not have any information about the wave patterns arising in the solution. Therefore we have to use a central approximation of the state at the interface. Such an approximation is used in the Richtmayer scheme [21]. The state at $t=t^{j}+\frac{\Delta t}{2}, x=0$ can be approximated just in terms of $u_{L}$ and $u_{R}$ by

$$
u_{I}=\frac{1}{2}\left(u_{R}+u_{L}\right)-\frac{\Delta t}{2 \Delta x}\left(f\left(u_{R}\right)-f\left(u_{L}\right)\right) .
$$

This appears to be a sort of a half time step of a shifted Lax-Friedrichs method, but in fact it is an exact expression of $\int_{-\frac{\Delta x}{2}}^{\frac{\Delta x}{2}} u\left(t^{j}+\frac{\Delta t}{2}, x\right) d x$. Thus if the time step $\Delta t$ is chosen according to a locally sharp CFL condition, the state $u_{I}$ is a reasonable approximation of the state at the interface.

The backward fluctuations of the Lax-Friedrichs method are given by

$$
D^{-}=\frac{1}{2}\left(f\left(u_{R}\right)-f\left(u_{L}\right)\right)-\frac{\Delta x}{2 \Delta t}\left(u_{R}-u_{L}\right) .
$$

For a general flux function $f$ and a $\Delta t$ corresponding to a CFL number $C<1$ this expression is only zero if $u_{L}=u_{R}$. Therefore we have to relax (11) into the minimization problem (12).
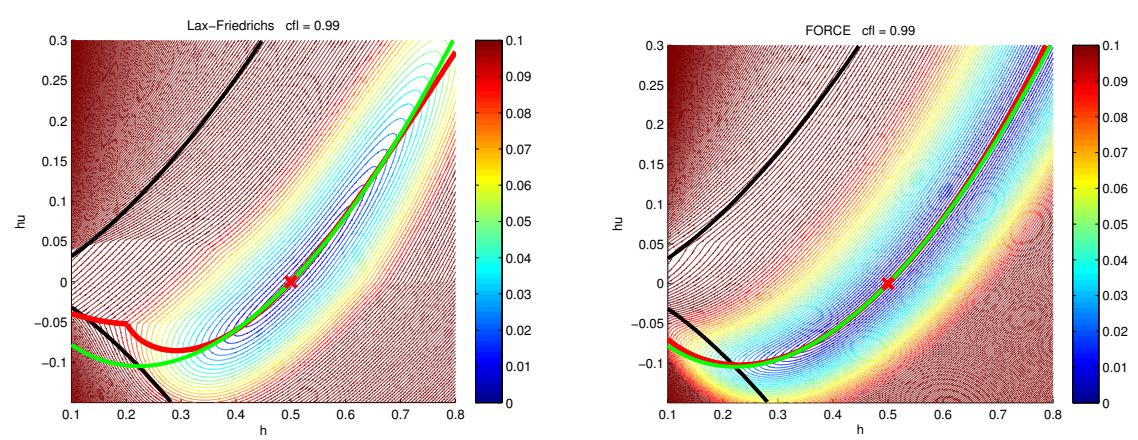

Figure 3: The value of $\left\|D^{-}\left(u_{L}, u_{R}\right)\right\|_{2}$ for $u_{R}=\left(\frac{1}{2}, 0\right)^{T}$ and varying $u_{L}$ for the Lax-Friedrichs method (left) and the FORCE method (right).

On the left side of Figure 3 the values of $\left\|D^{-}\left(u_{L}, u_{R}\right)\right\|_{2}$ for the shallow water equations (21) are shown with a fixed value $u_{R}=\left(\frac{1}{2}, 0\right)^{T}$ and varying $u_{L}$. These values are only small locally around $u_{R}$. Nevertheless this potential is orientated along the exact Lax-curve (green). Further, by looking at the minimum w.r.t. $q$ for every fixed value of $h_{L}$, we obtain the magenta curve which locally provides a reasonable approximation to the Lax-curve. The distance of these two curves is shown in Figure 4 on the left side. On the right side the values of $\left\|D^{-}\left(u_{L}, u_{R}\right)\right\|_{2}$ along the magenta curve are shown. These values indicate 

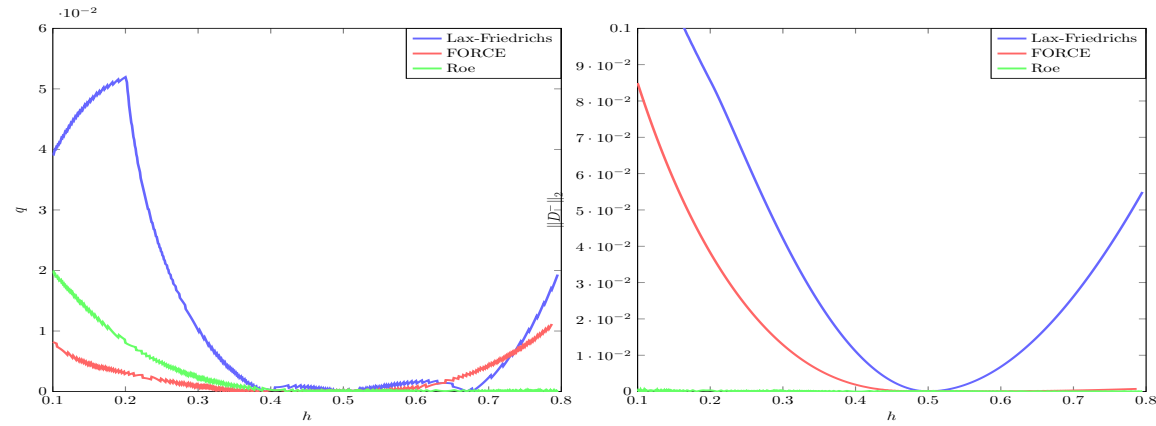

Figure 4: Lax-Friedrichs method: The values of $\left\|D^{-}\left(u_{L}, u_{R}\right)\right\|_{2}$ along the green and the black curve of figure 3.

the strength of the left going waves we have to admit due to the switching from (11) to (12). Locally around $u_{R}$ the values of $\left\|D^{-}\left(u_{L}, u_{R}\right)\right\|_{2}$ are small, but they increase quadratically with the distance to $u_{R}$. Thus when solving a Riemann Problem with a big initial jump we do expect at the first few time steps some contributions of left going waves, but the closer we come to the stationary state at the interface these waves disappear.

\subsection{FORCE solver}

The FORCE solver is a monotone central solver [22, 21] and we do not have any information about the orientation or structure of single waves. Therefore we follow a similar approach as for the Lax-Friedrichs method and we use (15) as an approximation of $u_{I}$.

The backward fluctuations of the FORCE scheme at the junction are

$$
D_{F O R C E}^{-}=\frac{1}{2}\left(F_{1 / 2}^{L F}+f\left(u_{I}\right)\right)-f\left(u_{L}\right),
$$

where $u_{I}$ is the approximation of the state at the interface given by formula (15). As before we display the values of $\left\|D^{-}\left(u_{L}, u_{R}\right)\right\|_{2}$ with $u_{R}=\left(\frac{1}{2}, 0\right)^{T}$ in figure 3. The magenta line indicates the minimum w.r.t. $q$ for each value of $h_{L}$. This curve is a good approximation to the actual Lax-curve. Its distance is shown on the left side of Figure 4 . On the right side we observe that the value of $\left\|D^{-}\left(u_{L}, u_{R}\right)\right\|_{2}$ is smaller along the Hugoniot loci than along the integral curve, but always better than for the Lax-Friedrichs scheme.

\section{Solving a constraint minimization problem}

In this section a procedure to solve a constraint minimization problem is discussed. We are interested in a solution of the problem

$$
\begin{aligned}
& \min _{u_{L} \in \mathbb{R}^{m \cdot n}}\left\|D^{-}\left(u_{L}, u_{R}\right)\right\|_{2}^{2} \\
& \text { s.t. } \Phi\left(u_{I}\left(u_{L}, u_{R}\right)\right)=0,
\end{aligned}
$$


where $u_{L}$ is a vector of states in the ghost cells, $u_{R}$ are the given values inside the domain, $u_{I}$ is the approximation of the state at the interface, $\Phi$ are the coupling conditions and $D^{-}$the left going fluctuations according to the numerical scheme applied.

To solve this problem we set up the following Lagrange functional

$$
L\left(u_{L}, \mu\right)=g\left(u_{L}\right)+\mu^{T} \tilde{\Phi}\left(u_{L}\right)
$$

with the functions $g\left(u_{L}\right)=\left\|D_{1 / 2}^{-}\left(u_{L}, u_{R}\right)\right\|_{2}^{2}$ and $\tilde{\Phi}\left(u_{L}\right)=\Phi\left(u_{I}\left(u_{L}, u_{R}\right)\right)$. In a local minimum its derivatives w.r.t. $u_{L}$ and the Lagrange multiplier $\mu \in \mathbb{R}^{c}$ should vanish, i.e.

$$
\begin{aligned}
\nabla_{\mu} L^{T}[\nu]=\nu^{T} \tilde{\Phi} & =0 & & \forall \nu \in \mathbb{R}^{c} \\
\nabla_{u_{L}} L^{T}[\xi]=\nabla_{u_{L}} g^{T} \xi+\mu^{T} \nabla_{u_{L}} \tilde{\Phi} \xi & =0 & & \forall \xi \in \mathbb{R}^{m \cdot n} .
\end{aligned}
$$

The first equation just states the coupling conditions, the second one is an overdetermined linear system for $\mu$, since $c<m \cdot n$. Its minimizing solution is given by

$$
\mu=-\left(\nabla_{u_{L}} \tilde{\Phi} \nabla_{u_{L}} \tilde{\Phi}^{T}\right)^{-1} \nabla_{u_{L}} \tilde{\Phi} \nabla_{u_{L}} g
$$

Finally for an update of a given value $u_{L}^{(i)}$ we apply

$$
\begin{aligned}
\tilde{u}_{L}^{(i+1)} & =u_{L}^{(i)}-\alpha \nabla_{u_{L}} L\left(u_{L}^{(i)}, \mu\right) \\
& =u_{L}^{(i)}-\alpha\left(\nabla_{u_{L}} g+\nabla_{u_{L}} \tilde{\Phi}^{T} \mu\right),
\end{aligned}
$$

where $\mu$ is computed according to (17) and $\alpha$ is some step size, e.g. determined by the Armijo rule [2]. The value $\tilde{u}_{L}^{(i+1)}$ we use as initial guess of the nonlinear solver for $\tilde{\Phi}\left(u_{L}^{(i+1)}\right)=0$. This second step assures that the solution fulfills the coupling conditions.

In the numerical examples of section 6 we use the above procedure for all the solvers presented in section 4 . Especially we do not apply any simplifications although they might increase the performance of the solvers. We stop the iteration (18) after 100 steps or if the change in the relative gradient is less than 1.e -7 . Both values appear to be too narrow for the considered problems, but the efficient numerical treatment of a minimization problem is not in the main focus of the present paper.

\section{Numerical tests}

In this section we study different test cases considering various aspects of the numerical schemes presented in section 4 . First we present a set of examples considering the shallow water equations, investigating the accuracy, convergence and deviation to the classical solvers at a 1-to-1 junction. Also tests including 
different numbers of connected edges are shown. Finally a three way junction for the full Euler equations is considered, showing that also the interaction of multiple waves can be captured accurately.

In all numerical tests we fix the spatial domain to $[0,1]$ and it is either discretized it with 100 cells per edge or with 2000 cells for the convergence studies. The time step is chosen adaptively according to a CFL condition

$$
\Delta t=C \frac{\Delta x}{\lambda_{\max }} .
$$

Here $\lambda_{\max }$ is the maximum of the absolute values of the eigenvalues of $\nabla f$ and in all the test cases we used $C=0.9$. At the right ends of the domains free outflow boundary conditions are applied [18, 21]. The coupling conditions are solved using the algorithm described in section 5 .

\subsection{Shallow water equations}

In the following test cases we will consider a single junction conneting a varying number of edges. On each edge $k$ the states $u^{k}=\left(h^{k}, q^{k}\right)^{T}$ are governed by the shallow water equations

$$
\begin{aligned}
\partial_{t} h^{k}+\partial_{x} q^{k} & =0 \\
\partial_{t} q^{k}+\partial_{x}\left(\frac{\left(q^{k}\right)^{2}}{h^{k}}+\frac{1}{2}\left(h^{k}\right)^{2}\right) & =0 .
\end{aligned}
$$

$h$ denotes the height of water in an rectangular open channel and $q$ is the flow in $x$-direction. In the case of subcritical flow $\left|\frac{q}{h}\right|<\sqrt{h}$ the following set of coupling conditions is often used $[15,13]$

$$
\begin{aligned}
\sum_{k=1}^{m} q^{k} & =0 \\
h^{k}-h^{1} & =0 \quad \forall k=2, \ldots, m .
\end{aligned}
$$

Equation (22) assures the conservation of mass in the complete network and equations (23) state the equality of the heights at the node. In the numerical method we apply (8) on (23), but (10) on (22). Other possible choices of coupling conditions can be found in $[15,20,4,3,12]$.

In the following pictures only $h$ is shown, but in all the examples the behavior in $q$ is of an identical quality. Here we recall that all edges are connected at $x=0$, e.g. waves exiting the first edge on the left enter the other edges also on the left. The scopes of the picture are varying in order to focus on the relevant parts of the solutions.

\subsubsection{1-to-1 junction}

The simplest junction possible is a junction connecting just two identical edges. The advantage of this test case is that the solution coincides with the

solution on one single line of double length, where the first half corresponds to 

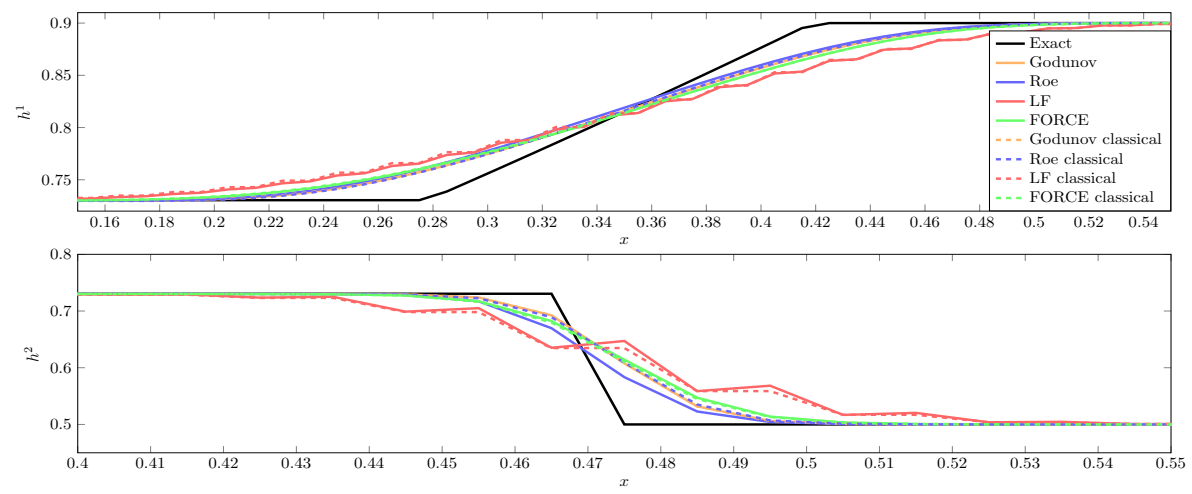

Figure 5: '1-to-1' junction: Comparison of the solvers with the classical ones.

the first edge and the second half corresponds to the second edge. This allows us to compare the solvers at the junction with the underlying classical methods.

In figure 5 the relevant parts of the exact and numerical solutions to a Riemann problem with $u^{1} \equiv(0.9,-0.1)^{T}$ and $u^{2} \equiv(0.5,0)^{T}$ at time $t=0.5$ are plotted. The dashed lines indicate the solutions obtained using the classical solvers on a continuous line, mapped onto the corresponding edges. The results of the junction solvers are not exactly identical to the classical ones, but of a comparable quality. On the left part of figure 6 the convergence of the values
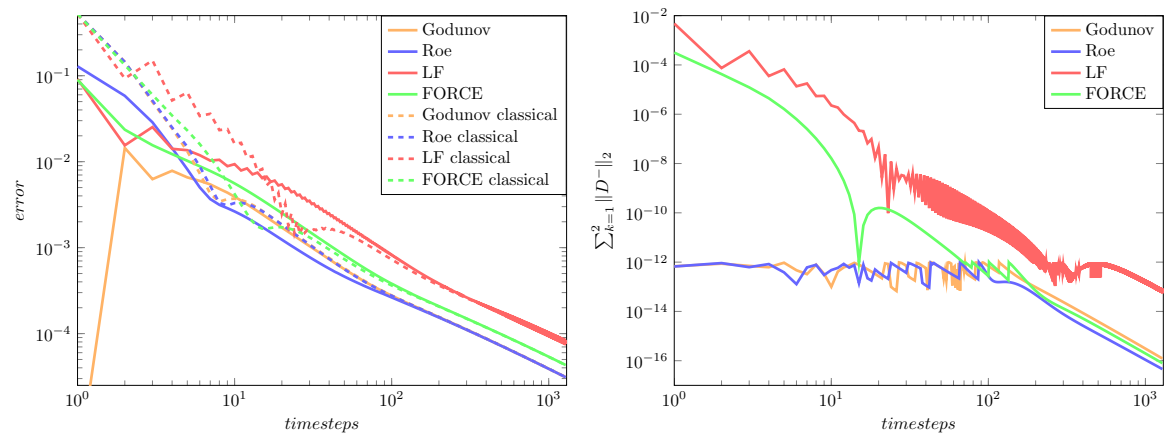

Figure 6: '1-to-1' junction: Left: Convergence to the exact solution. Right: Total strength of left going waves.

in the ghost cells towards the exact solution at the junction are shown, i.e. the values $\sum_{k=1}^{2}\left\|u_{L}^{k}-u_{\text {exact }}^{k}(x=0)\right\|_{2}$ are plotted versus the number of time steps. The switching from 100 to 2000 cells does not effect the output of the solver at the junction, since the CFL number is kept constant. We can see that the solutions of the junction solvers converge in the same manner as their classical counterparts do. On the right side of figure 6 the strength of the left going fluctuations at the node is plotted. For the central schemes the value of 
$\sum_{k=1}^{2}\left\|D^{-}\right\|_{2}$ is small right from the beginning and decaying as the solutions converge to the stationary state. For the Godunov and the Roe scheme it is in the range of the accuracy of the nonlinear solver for the coupling conditions. Here we emphasis that the value of $\sum_{k=1}^{2}\left\|D^{-}\right\|_{2}$ does not indicate a loss of mass. The total mass in the system only varies in the range of the accuracy of the nonlinear solver for the coupling conditions, i.e it does not exceed $10^{-14}$ in the considered time period. In terms of accuracy no relevant difference to the classical solvers van be observed, just the numerical effort is much higher.

\subsubsection{Three way junction}

In this test case we investigate a three way junction $m=3$. At first we consider a Riemann problem consisting of the initial data $u^{1}=(0.9,0)^{T}, u^{2}=$ $(0.5,0)^{T}$ and $u^{3}=(0.3,0)^{T}$.
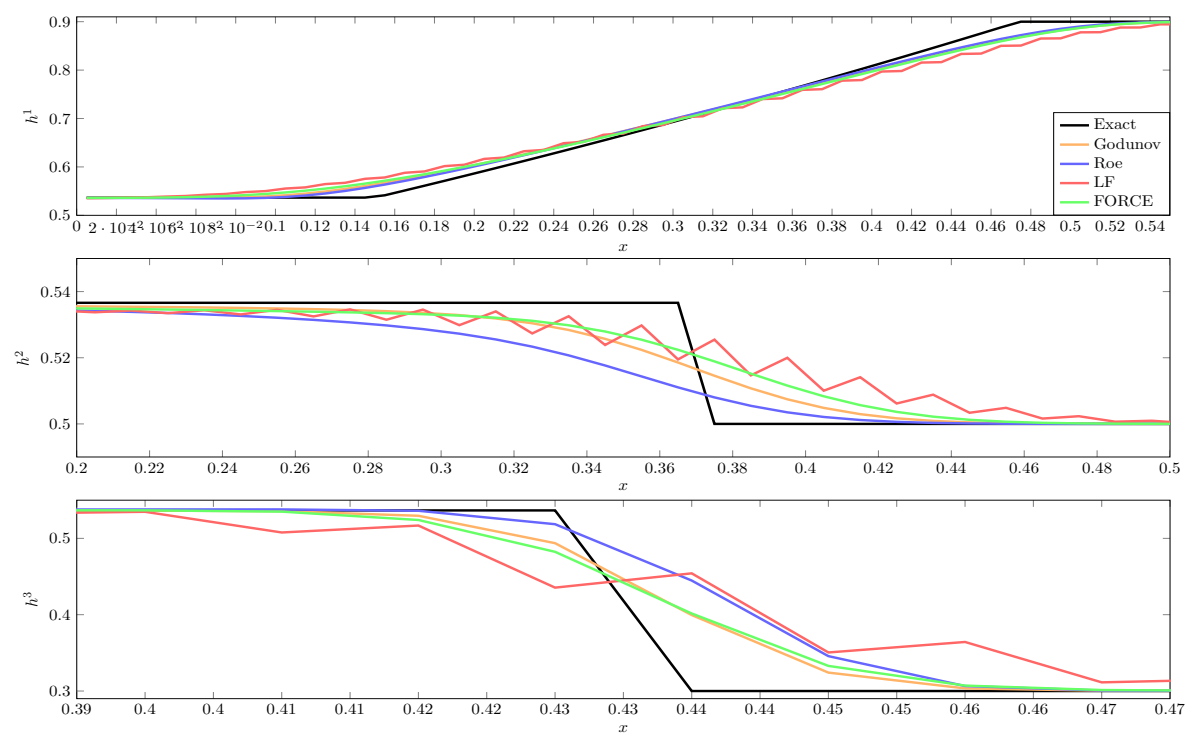

Figure 7: 'Three-way' junction: Solution of a Riemann problem.

In Figure 7 the interesting parts of the solutions are shown. All numerical schemes are able to capture the waves emerging from the junction. The quality of the approximation resembles the accuracy of the underlying original methods. The Lax-Friedrichs method tends to show some wiggles, but a similar behavior can also be observed for the classical scheme [8] and is not to be confused with destabilizing oscillations.

In Figure 8 on the left side we show the convergence of the values in the ghost cells towards the exact state at the junction, i.e. the value $\sum_{k=1}^{3} \| u_{L}^{k}-u_{\text {exact }}^{k}(x=$ $0) \|_{2}$ is plotted. The dashed lines indicate the slopes for first order convergence. After some initial variations all schemes converge with order one towards the exact states. In the first step the Godunov method captures the exact values 

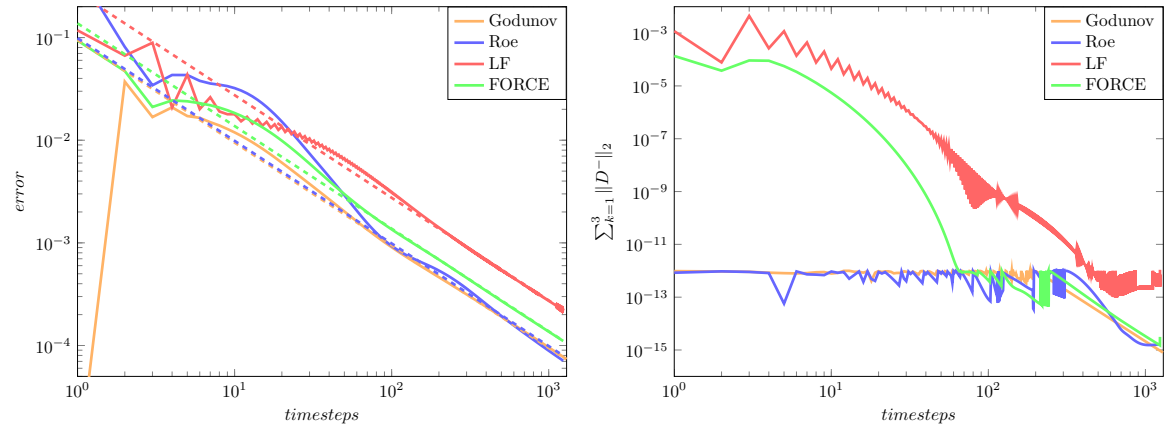

Figure 8: 'Three-way' junction: Left: Convergence rates. Right: Strength of left going waves.

in the ghost cells, but this advantage is immediately lost since the states $u_{R}$ vary in each iteration. The strength of the left going fluctuations $\sum_{k=1}^{3}\left\|D^{-}\right\|_{2}$ is shown on the right hand side. For the central schemes it decays with the number of time steps, whereas the Godunov and Roe method stay within the tolerance of the nonlinear solver of the coupling conditions.

As final test for the three-way junction, we check that the solvers can be applied to an arbitrary set of subsonic states at the junction. Therefore we fix the initial states in the second and third edge by $u^{2}=(0.5,0)^{T}$ and $u^{3}=$ $(0.75,0)^{T}$. The initial state in the first edge varies from $0.1 \leq h \leq 1.2$ and $-1 \leq q \leq 1$ with a sampling width 0.025 in each direction. The supersonic initial states are excluded in this test.

For each such initial data we compute the relative error at the junction $\sum_{k=1}^{3} \frac{\left\|u_{L}^{k}-u_{\text {exact }}^{k}(x=0)\right\|_{2}}{\left\|u_{\text {exact }}(x=0)\right\|_{2}}$ at time $T=0.1$ on a grid of 100 cells per edge. This corresponds to the relative error at the junction after about 10 iterations. In figure 9 the errors of the different schemes are shown in the state space. We observe that for all initial data the schemes do converge towards the exact solution. Near the supersonic boundary the quality of the approximation decays, whereas for smaller waves the approximation is best. As we already have seen before, the quality of the approximation also depends on the choice of the solver, but in all cases it is within an acceptable range.

\subsubsection{Five way junction}

As final example of the shallow water equations we consider a junction connecting five edges. This test confirms that the numerical solvers can also handle junctions connecting a larger number of edges. The results in Figure 10 do not differ much from those of the previous test cases. All numerical methods can capture the involved waves. For smaller waves the resolution appears to be worse, which is mainly caused by the scales considered.

In Figure 11 the convergence to the exact solution and the strength of the left going waves at the junction are shown. The quality of both quantities is not effected by the number of junctions involved. 

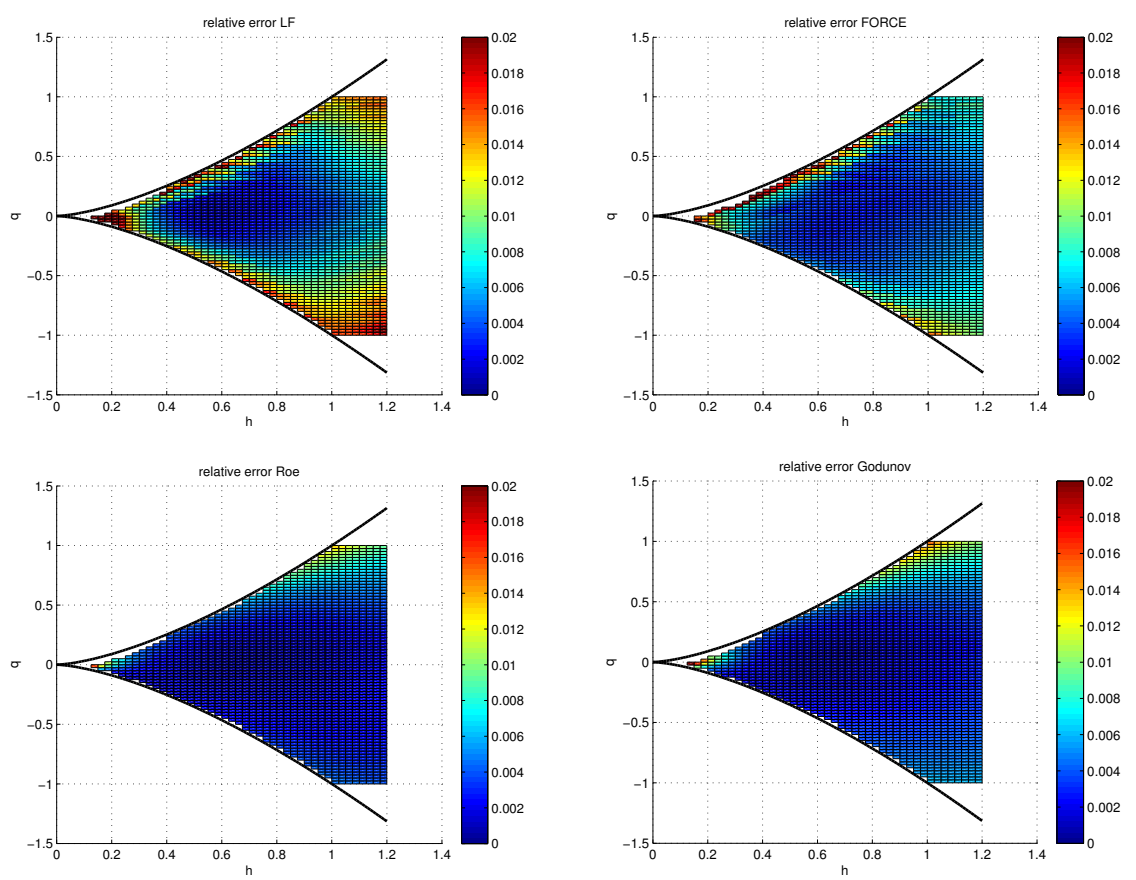

Figure 9: 'Three-way' junction: Testing the state space in edge 1 for the Lax-Friedrichs (upper left), FORCE (upper right), Roe (lower left) and Godunov (lower right) method.

Thus the main difference of the test case with five edges is the increase of computational effort. Since the dimensions of the minimization problem and the coupling conditions therein increased, more iterations are necessary to reach accurate results.

\subsection{Euler equations}

In this test case we consider a junction which connects three edges governed by Euler equations. With this example we check if the numerical methods are also capable to treat Riemann problems at the junction, which involve multiple waves entering one single edge. The Euler equations are given by

$$
\begin{aligned}
\partial_{t} \rho^{k}+\partial_{x} q^{k} & =0 \\
\partial_{t} q^{k}+\partial_{x}\left(\frac{\left(q^{k}\right)^{2}}{\rho^{k}}+p^{k}\right) & =0 \\
\partial_{t} E^{k}+\partial_{x}\left(\frac{q^{k}}{\rho^{k}}\left(E^{k}+p^{k}\right)\right) & =0,
\end{aligned}
$$

with the additional closure relation $p^{k}=(1.4-1)\left(E^{k}-\frac{\left(q^{k}\right)^{2}}{2 \rho^{k}}\right)$. 

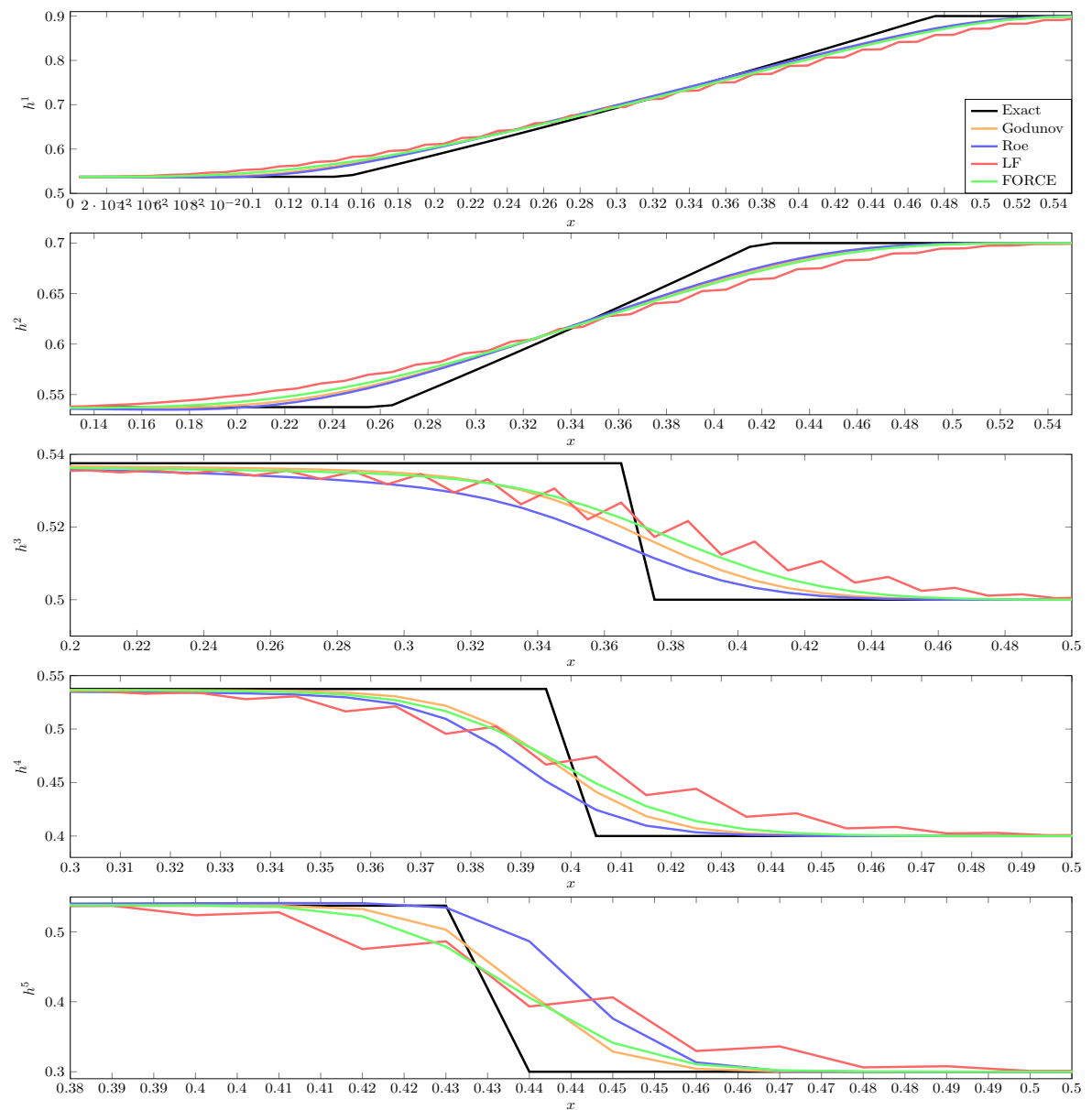

Figure 10: 'Five-way' junction: solution of a Riemann problem.

For the coupling of Euler equations there exists no general set of coupling conditions. Thus we chose an natural extension of the previous conditions (23) and (22) by

$$
\begin{aligned}
\sum_{k=1}^{m} q^{k} & =0 \\
\rho^{k}-\rho^{1} & =0 \quad \forall k=2, \ldots, m \\
\sum_{k=1}^{m} \frac{q^{k}}{\rho^{k}}\left(E^{k}+p^{k}\right) & =0 .
\end{aligned}
$$

The first equation conserves the total mass in the network, the following $m-1$ force the densities to coincide at the node. The last equation establishes the conservation of energy in the whole system. 

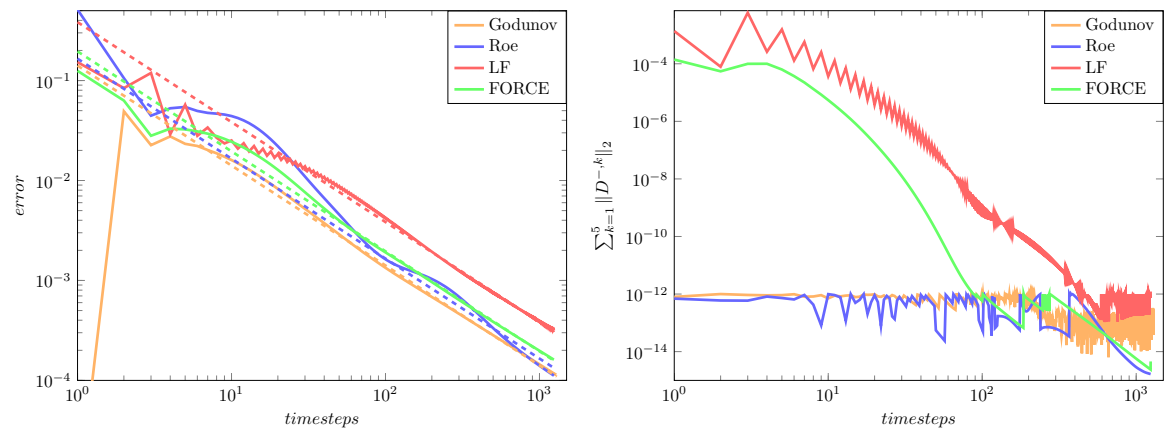

Figure 11: 'Five-way' junction: Left: Convergence to the exact solution at the junction. Right: Total strength of left going waves.

This choice of coupling conditions directly fixes the number of incoming and outgoing characteristics at the node we are allowed to consider. In the first edge two characteristics enter the domain, but in the other two edges only one wave moves to the right in each of them. As corresponding initial data we consider a Riemann problem at the junction given by

$$
u^{1}=(0.5,0.1,1.27)^{T}, \quad u^{2}=(0.7,-0.14,1.77)^{T}, \quad u^{3}=(1.0,-0.2,2.52)^{T} .
$$

The spatial domain and the numerical setting is chosen identical to the previous examples, the final time is $T=0.4$.
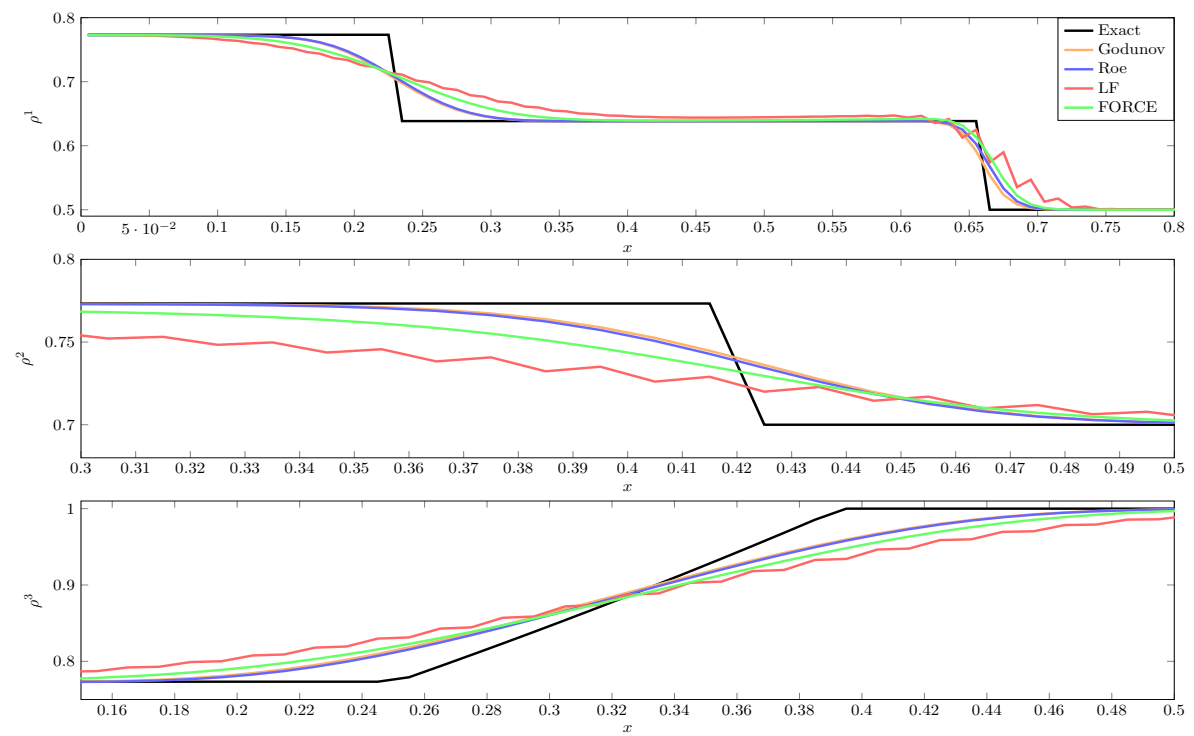

Figure 12: 'Three-way' Euler junction: solution of a Riemann problem. 
Figure 12 shows relevant parts of the solution in the edges. In the pictures only $\rho$ is shown, but the quality of the results is comparable in the other components. We can see that the states at the junction as well as all the involved waves are captured by the numerical methods. The particular behavior of the solver inside the domain is not altered by the procedure at the node, e.g. all solvers have difficulties in resolving the contact discontinuity.
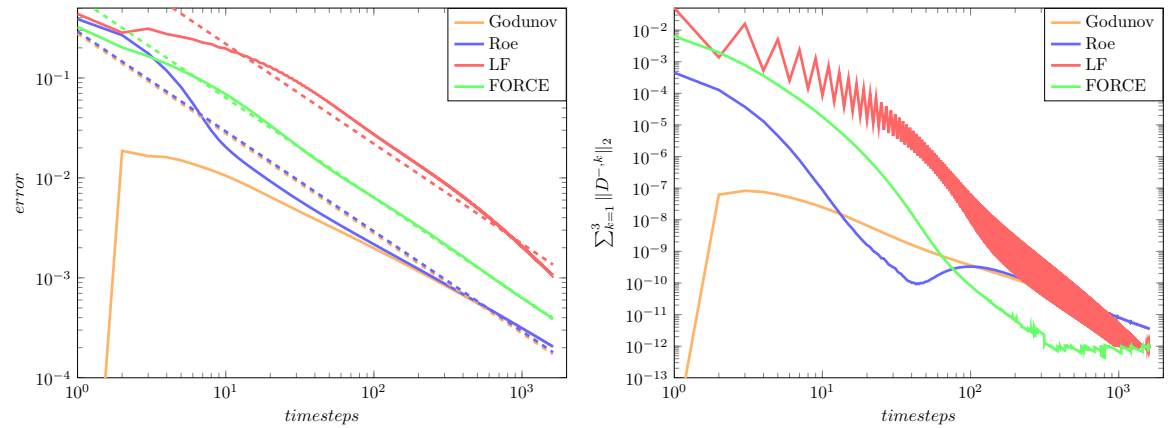

Figure 13: 'Three-way' Euler junction: Left: Convergence to the exact states at the junction. Right: Total strength of left going waves.

In Figure 13 the convergence of the value in the ghost cells towards the exact state at the interface is shown. All three schemes converge with order one to the exact solution. On the right half the total strength of the left going waves $\sum_{k=1}^{3}\left\|D^{-, k}\right\|_{2}$ at the junction is plotted. Here it is important to note that both, Godunov and Roe scheme, do have contributions above the accuracy of the nonlinear solver for the coupling conditions. This just shows that the algorithm presented in section 5 is not capable to exactly solve the minimization problem as the set of admissible states has a complicated shape. This behavior can be easily cured by skipping the formulation (12) and returning to (11) for these solvers. It also decreases the numerical effort remarkably. For the central solvers such a modification is not possible, but the strength of their left going waves decays with the number of iteration.

\section{Conclusions}

In this paper we have developed a procedure to extend classical solvers for hyperbolic conservation laws to networks of hyperbolic conservation laws. This method is especially suitable for systems, where the detailed structure of the Riemann problem can not be provided explicitly. We observed that the accuracy is comparable with the common Godunov solver, but for central methods the effort at the junction increases remarkably. In most cases the Roe solver appears to be the best choice, but if a central scheme is needed the FORCE scheme is a reasonable alternative. 


\section{Acknowledgements}

The author would like to thank Jochen Kall, Oliver Tse and Claudia Totzeck for hints and helpful discussions.

[1] M.K. Banda, M. Herty, A. Klar, Coupling conditions for gas networks governed by the isothermal Euler equations, Netw. Heterog. Media 1 (2006) 295-314.

[2] J.F. Bonnans, J.C. Gilbert, C. Lemaréchal, C.A. Sagastizábal, Numerical optimization, Universitext, second ed., Springer-Verlag, Berlin, 2006. Theoretical and practical aspects.

[3] R. Borsche, R.M. Colombo, M. Garavello, On the coupling of systems of hyperbolic conservation laws with ordinary differential equations, Nonlinearity 23 (2010) 2749.

[4] R. Borsche, R.M. Colombo, M. Garavello, Mixed systems: Odes - balance laws, Journal of Differential Equations 252 (2012) 2311-2338.

[5] R. Borsche, J. Kall, Ader schemes and high order coupling on networks of hyperbolic conservation laws, Journal of Computational Physics 273 (2014) $658-670$.

[6] R. Borsche, A. Klar, S. Kühn, A. Meurer, Coupling traffic flow networks to pedestrian motion, Mathematical Models and Methods in Applied Sciences 24 (2014) 359-380.

[7] G. Bretti, R. Natalini, B. Piccoli, Numerical algorithms for simulations of a traffic model on road networks, J. Comput. Appl. Math. 210 (2007) 71-77.

[8] M. Breuß, About the lax-friedrichs scheme for the numerical approximation of hyperbolic conservation laws, PAMM 4 (2004) 636-637.

[9] J. Brouwer, I. Gasser, M. Herty, Gas pipeline models revisited: model hierarchies, nonisothermal models, and simulations of networks, Multiscale Model. Simul. 9 (2011) 601-623.

[10] M.J. Castro, J.A. García-Rodríguez, J.M. González-Vida, J. Macías, C. Parés, M.E. Vázquez-Cendón, Numerical simulation of two-layer shallow water flows through channels with irregular geometry, J. Comput. Phys. 195 (2004) 202-235.

[11] R.M. Colombo, M. Garavello, A well posed Riemann problem for the $p$ system at a junction, Netw. Heterog. Media 1 (2006) 495-511.

[12] R.M. Colombo, M. Garavello, Comparison among different notions of solution for the $p$-system at a junction, Discrete Contin. Dyn. Syst. (2009) 181-190. 
[13] M.Á. Fernández, V. Milišić, A. Quarteroni, Analysis of a geometrical multiscale blood flow model based on the coupling of ODEs and hyperbolic PDEs, Multiscale Model. Simul. 4 (2005) 215-236 (electronic).

[14] M. Garavello, B. Piccoli, Traffic flow on networks, volume 1 of AIMS Series on Applied Mathematics, American Institute of Mathematical Sciences (AIMS), Springfield, MO, 2006. Conservation laws models.

[15] G. Kesserwani, R. Ghostine, J. Vazquez, R. Mosé, M. Abdallah, A. Ghenaim, Simulation of subcritical flow at open-channel junction, Advances in Water Resources (2008) 287-297.

[16] O. Kolb, J. Lang, P. Bales, An implicit box scheme for subsonic compressible flow with dissipative source term, Numer. Algorithms 53 (2010) 293-307. URL: http://dx.doi.org/10.1007/s11075-009-9287-y. doi:10.1007/s11075-009-9287-y.

[17] A.S. Leon, Improved Modeling of Unsteady Free Surface, Pressurized and Mixed Flows in Storm-sewer Systems, Ph.D. thesis, University of Illinois at Urbana-Champaign, 2007.

[18] R.J. LeVeque, Finite Volume Methods for Hyperbolic Problems, Cambridge Texts in applied mathematics, 2002.

[19] L.O. Müller, E.F. Toro, A global multiscale mathematical model for the human circulation with emphasis on the venous system, International Journal for Numerical Methods in Biomedical Engineering (2014) n/a-n/a.

[20] G.A. Reigstad, Numerical network models and entropy principles for isothermal junction flow, Networks and Heterogeneous Media 9 (2014) 65 -95 .

[21] E.F. Toro, Riemann solvers and numerical methods for fluid dynamics: a practical introduction, Springer, 2009.

[22] E.F. Toro, A. Hidalgo, M. Dumbser, Force schemes on unstructured meshes i: Conservative hyperbolic systems, Journal of Computational Physics 228 (2009) 3368-3389. 